\title{
Experimental results of time-frequency domain characters for divers with open-circuit SCUBA
}

\author{
Zhang Jiarong $^{1}$, Li Hailong $^{1}$, Zhang Xiaoliang ${ }^{2, a}$ \\ ${ }^{1}$ Systems Engineering Research Institute, Beijing, China \\ ${ }^{2}$ Science and Technology on Underwater Acoustic Antagonizing Laboratory, Systems Engineering \\ Research Institute \\ azxl.seri@163.com
}

Keywords: diver detection; target strength; time-frequency domain characters

\begin{abstract}
In this paper, we studied the time-frequency domain characters of divers in lake trail and proposed some experimental results. The diver we invited in the trail was equipped with open-circuit SCUBA. We recorded the breathing sound of the diver in different motion state and analyzed its characters both in time and frequency domain. We extracted some key characteristics and practical parameters for the diver detection and identification. The results we obtained from the trail support the passive diver detection.
\end{abstract}

\section{Introduction}

Marine infrastructure such as bridges, power plants, port, and harbor facilities etc. are very costly assets of any country and are vulnerable to attacks from adversaries and terrorist organizations. A threat to these facilities can come from underwater submersibles and divers. Thus, early detection and persecution of these underwater threats is very important. Divers are listed as the primary goal of underwater anti-terrorism by many countries for their high destructiveness [1-2]. Diver detection has many approaches, such as the infrared detection, radar detection, vision detection, acoustic detection and et al. Acoustic detection, including active and passive means, is considered as the most effective way.

In passive means, characters of the breathing sound are the main basis for diver recognition, and envelop spectrum analysis is the most common used approach [3]. Characters of the breathing sound are related to the SCUBA types (open-circuit or close-circuit) and the diver's motion state [4-5]. In using envelop spectrum approach to analyze the breathing sound characters, different frequency band signal components and different breathing cycles has different contributions to the result, but it was rarely discussed in the published literatures.

In this paper, we address the problem of extracting key characters and practicable parameters for diver detection. A diver equipped with wet suit and open-circuit SCUBA was took as the study object and lake trail was carried out at Songhua Lake in Jilin Province, China. In trail, we recorded the diver's breathing sound while the diver was in different motion state. These data were well processed and the results are proposed in detail.

\section{Experiment Setup}

Figure 1 (a) shows the general location of the area where the experiment was done. Depth of the lake at this position is about 30 meters. The diver who was $175 \mathrm{~cm}$ tall and weighed $80 \mathrm{~kg}$ was used as a target. He was wearing a $5 \mathrm{~mm}$ thick nylon wetsuit and had an open-circuit scuba with $12 \mathrm{~L}$ large air tank which was made of aluminum alloy. The whole experiment course consisted of two main stages: breathing sound collection on different motion state, and max detection range measurement. Fig.1 (b) shows the schematic of the first stage, the diver kept underneath the water at about 3 meters deep and swam around the experiment boat on different speed. Fig.1 (c) shows the schematic of the second stage, the diver swam from the experiment boat towards the distance. 


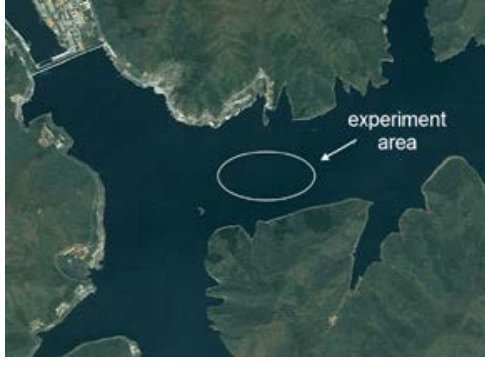

(a)

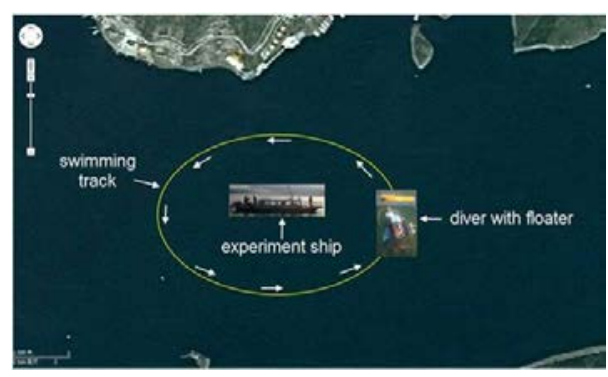

(b)

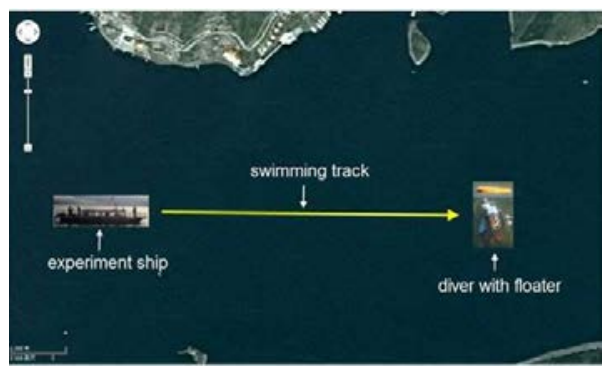

(c)

FIG. 1. Experiment setup.

\section{Breathing Sound Characters Analyzing}

\section{Waveform envelop features}

Fig.2 (a-c) shows the waveform envelop of the original and filtered signal acquired in the first trail stage. Envelope of the original signal is shown in Fig.2 (a), as we can see, breathing rhythm can hardly be distinguished. The breathing sound consists of expiration bubbles and regular vibrations, in order to investigate the features of these two different components, we processed the recorded breathing sound by follow steps. First, we filtered the raw signal use band-pass filter in different frequency bands and replayed the filtered signal, the results shows that energy of the expiration bubbles is mainly concentrated in the low frequency band (below $2 \mathrm{kHz}$ ) while the regulator vibration is mainly in high frequency band (over $2 \mathrm{kHz}$ ). Followed by this result, the second step processing was conducted to observe the envelop features of these two components. We separately filtered the raw signal use two different frequency bands: $0.01-2 \mathrm{kHz}$ and $3-10 \mathrm{kHz}$, and the waveform envelopes are shown in Fig.2 (b) and (c). Obviously, periodicity is much more distinct in high frequency band than that in low frequency band. This result indicates that the high band is the ideal choice for diver passive detection. From Fig.2 (c), some other conclusions can also be drew: first, every breathing cycle lasts about 3 seconds; second, there is some rest energy of expiration bubbles in high frequency band, though its main part is in low frequency band. As Fig.2 (c) shows, in every breathing cycle, the lower part is the expiration bubble and the higher part is the regulator vibration.

In the second trail stage, we measured the furthest available monitoring distance is about 70 meters, without using any sophisticated signal processing algorithm. During the course of the diver swam from near to far, energy attenuation of the expiration bubbles (at low frequency band) is much quicker than that of the regulator vibration (at high frequency band). At the distance of 70 meters, only the regulator vibration noise can be monitored. Waveform envelopes of the original and filtered signal at the furthest distance are shown in Fig.2 (d-f). Waveform envelopes of the original and low frequency band signal are totally submerged in the background noise as Fig.2 (d-e) shows, only the high frequency band signal has tiny protuberances as Fig.2 (f) shows. This result demonstrates that the high band is the ideal choice for diver passive detection in another aspect. 

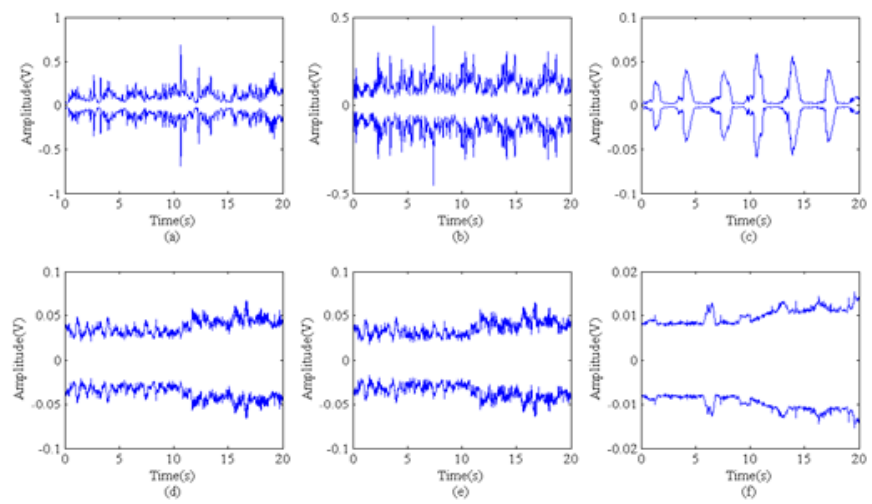

FIG. 2. Waveform envelops.
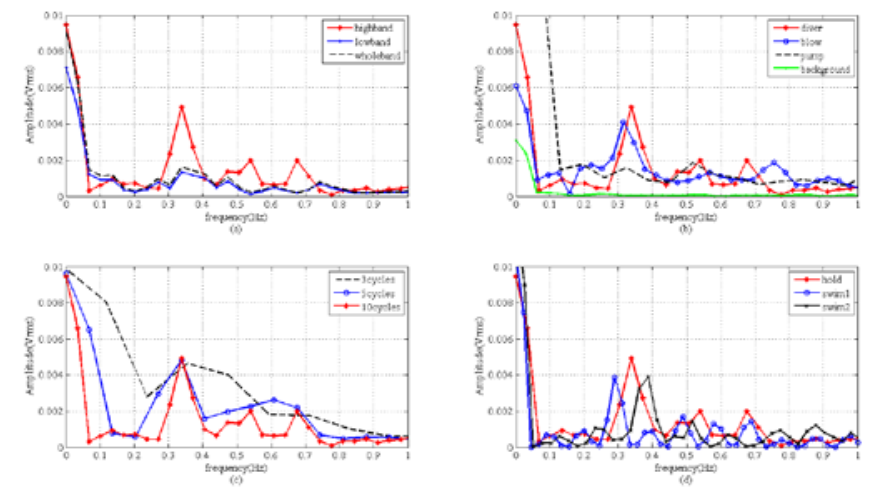

FIG. 3. Target characters in different conditions.

\section{Target characters}

Target characters of the breathing sound were observed using waveform envelop spectrum, steps are as [6] shows, results of different processing conditions are shown in Fig.3.

Firstly, we observe the best filter band to detecting the diver. According to the result as Fig.2 shows, three bands were chosen: low band (0.01-2 kHz), high band (3-10 kHz) and whole band (original signal without filtering). Breathing sound were filtered by band-pass filter of these bands, and their envelop spectrum curves are shown in Fig.3 (a) marked with "lowband", "highband" and "wholeband". As we can see, there is an obvious peak of the "highband" curve between 0.3 and 0.4 $\mathrm{Hz}$ (diver's breathing rate is in this range). This result makes the high band believed to be the ideal choice for diver detecting.

Secondly, we observe the envelop spectrum features of different sources. For comparison, four sources of sound were invited: diver breathing sound, man blowing sound, pump pumping sound and background noise. Blowing sound was used to simulate the diver's breathing sound, one of our staff blew the air to the water through a tube, and kept the likely breathing rhythm to divers. Pumping sound is a continuous sound, we used a pump to pump the air to water through a tube to generate it. 20 cycles of each source signal were chosen and filtered with band pass filter at 3-10 kHz band, results are shown in Fig.3 (b) marked with "diver”, "blow”, "pump” and "background”. Obviously, the "diver" curve and the "blow" curve have an obvious peak between $0.3 \mathrm{and} 0.4 \mathrm{~Hz}$ which is match to the diver's breathing rate. While the "pump" and "background" curves do not have this character. Hence, this character can be treated as an important evidence for the diver identifying.

Thirdly, we observe the best cycles (or signal length) for diver detecting. For comparison, we separately chosen 3 different cycles $(3,5,10)$ of breathing sound to process, results are as Fig.3 (c) shows marked with " 3 cycles", " 5 cycles" and " 10 cycles". Comparing the curves, we can find that around $0.33 \mathrm{~Hz}$ each one has a peak, the more cycles we chose to processing the sharper peak we can get. As a result, when using envelop spectrum to analyze the frequency domain characters of the diver's breathing sound, the more cycles the better, practically, 10 cycles is enough to obtain a 
reasonable result.

Finally, we observe the influence of motion activities to target characters. For comparison, breathing sound at three different states were chosen for processing, the three states were hold still, swam at low speed and swam at high speed, results are shown in Fig.3 (d) with corresponding curves marked with "hold", "swim 1" and "swim 2". Filter band we used here is 3-10 kHz and signal cycles is 10. As the figure shows, each curve has an obvious peak between 0.3 and $0.4 \mathrm{~Hz}$, and the position is related to the diver's motion activities. The peak position of "swim 2" curve is on right side of the "hold" curve, that's because when the diver swim at a high speed, its breathing rhythm is faster than that at hold state. But when the diver swim slowly, his breathing needs to cooperate with his actions, the breathing rhythm is slower than that at hold state, and its peak position is on left side of the "hold" curve. That means peak location is not a constant, it is varying with the motion activity changes, but the range is between 0.3 and $0.4 \mathrm{~Hz}$.

\section{Summary and Conclusions}

In this paper, lake trail was conducted to investigate the time-frequency characters of diver detection. A diver equipped with wetsuit and open-circuit SCUBA was took as the study object. The main purpose of this study is to draw some key characters and practicable parameters for diver detection. Some significant results are obtained as follows:

For the passive detection, $3-10 \mathrm{kHz}$ is the recommended frequency band for diver passive detection and the breathing cycle needs as much as possible, as a practical results, 10 cycles are enough to obtain a good performance.

\section{References}

[1] Trees C.C., Bissett, Paul W., Dierssen H., and et al.. Monitoring water transparency and diver visibility in ports and harbors using aircraft hyperspectral remote sensing. in proceedings of Photonics for Port and Harbor Security, March 2005, Orlando, FL, USA, pp.91-98.

[2] Basaran Y.H., Oktem M.H., Atabek Y. Bakim and C.E.. Diver detection sonar. in proceedings of 20th Signal Processing and Communications Applications Conference, April 2012, Fethiye, Mugla, Turkey, pp.1-2.

[3] Stolkin R., Sutin A., Radhakrishnan and et al.. Feature based passive detection of underwater threats. Proc.SPIE 2006(6204): 40-49.

[4] Chen X., Wang R. and Tureli U.. Passive acoustic detection of divers under strong interference. in proceeding of OCEANS 2006, Sep 2006, Boston, USA, pp.1-6.

[5] Chung K.W., Li H.B and Sutin A.. A Frequency-Domain Multi-Band Matched-Filter Approach to Passive Diver Detection. in proceeding of 41st Asilomar Conference on Signals, Systems and Computers, Nov 2007, Pacific Grove, CA, USA, pp.1-6.

[6] Stolkin R., Radhakrishnan S., Sutin A. and Rountree R.. Passive acoustic detection of modulated underwater sounds from biological and anthropogenic sources. in proceedings of Oceans 2007 MTS/IEEE Conference, Sep 2007, Vancouver, BC, Canada, pp.1-8. 